\title{
Excitation and trapping of lower hybrid waves in striations
}

\author{
N. Borisov ${ }^{1,2}$ and F. Honary ${ }^{1}$ \\ ${ }^{1}$ Lancaster University, Lancaster LA1 4WA, United Kingdom \\ ${ }^{2}$ Institute of Terrestrial Magnetism, Ionosphere and Radio Waves Propagation (IZMIRAN), \\ 142190 Troitsk, Russia
}

(Received 11 July 2008; accepted 5 November 2008; published online 3 December 2008)

\begin{abstract}
The theory of lower hybrid (LH) waves trapped in striations in warm ionospheric plasma in the three-dimensional case is presented. A specific mechanism of trapping associated with the linear transformation of waves is discussed. It is shown analytically that such trapping can take place in elongated plasma depletions with the frequencies below and above the lower hybrid resonance frequency of the ambient plasma. The theory is applied mainly to striations generated artificially in ionospheric modification experiments and partly to natural plasma depletions in the auroral upper ionosphere. Typical amplitudes and transverse scales of the trapped LH waves excited in ionospheric modification experiments are estimated. It is shown that such waves possibly can be detected by backscattering at oblique sounding in very high frequency (VHF) and ultra high frequency (UHF) ranges. (C) 2008 American Institute of Physics. [DOI: 10.1063/1.3035910]
\end{abstract}

\section{INTRODUCTION}

Low hybrid (LH) waves are often registered in space plasma. They are detected in the auroral upper ionosphere, ${ }^{1-4}$ at the Earth's magnetopause, ${ }^{5}$ in the vicinity of the Earth's magnetotail, ${ }^{6}$ at the dayside ionopause of Mars and Venus, ${ }^{7}$ near comets, ${ }^{8}$ etc. LH waves interact efficiently with electrons along the magnetic field lines and with ions across the magnetic field lines. Therefore they play significant role in different physical processes associated with particle acceleration, e.g., electron acceleration in the upstream region of the bow shock, ${ }^{9}$ solar flare ion and electron acceleration, ${ }^{10}$ transverse acceleration of ions in the Earth's ionosphere, ${ }^{1-3}$ etc.

One of the challenging problems related to $\mathrm{LH}$ waves is whether they are subjected to modulation instability and collapse. Despite the existing theoretical prediction in the twodimensional (2D) case $^{11,12}$ and in the three-dimensional (3D) case $^{13}$ there are no clear experimental confirmations that such a process indeed takes place in the Earth's ionosphere. Moreover, the data obtained by Freja satellite suggest that collapse of localized bursts of LH waves (spikelets) in the upper auroral ionosphere does not exist. ${ }^{4}$

Due to the important role of LH waves in physical processes they are actively investigated in laboratories with the help of special devices. ${ }^{14,15}$ Unfortunately, the existence of walls in chamber and specific ratio of the main parameters do not allow us to model accurately in laboratory the properties of LH waves relevant to space plasma. That is why a controlled experiment in space providing the exploration of $\mathrm{LH}$ waves properties and their dynamics would be of prime importance. Such an experiment can be carried out with the help of powerful high frequency (HF) radio waves acting on the ionospheric F-layer. This action results in different nonlinear processes. Many of them can be investigated experimentally by backscattering from the perturbed F-region in HF, VHF, and UHF ranges, or directly by rockets. Plasma and ion sound waves near the reflection height of the pump wave were observed by VHF and UHF radars situated rather close to the heating facility (see, e.g., Refs. 16-18). The experiments carried out at Troms $\varnothing$ and Arecibo made it possible to analyze the excitation of plasma and ion lines with high time or high spatial resolution and to find out interesting peculiarities of their development. In such a form this method can be applied for the investigations of irregularities which are not stretched along the magnetic field line. If the irregularities are strongly elongated with respect to the magnetic field (e.g., striations) the oblique sounding should be used to detect them. Many years ago such techniques was applied successfully at Platteville and Arecibo for the investigation of artificial small-scale irregularities. ${ }^{19-21}$ It was found that the cross section of backscattering at the radar frequency $f_{r}$ decreased with the frequency growth. ${ }^{21}$ Simultaneously the signals at the displaced frequencies $f_{r} \pm f_{h}$ ( $f_{h}$ is the heater frequency) were observed in the VHF and UHF ranges. ${ }^{20}$ Their cross section of backscattering increased with the radar frequency and achieved its maximum in the UHF range. At the same time such signals were absent in the HF range. At the beginning it was supposed that the signals at displaced frequencies appeared due to excitation of Langmuir waves. ${ }^{20}$ Later on it was concluded that at oblique sounding the elongated upper hybrid resonance (UHR) oscillations were responsible for the backscattered signals at the frequencies $f_{r} \pm f_{h}$. It is not surprising that such signals were observed only in the VHF and UHF ranges. According to the recent theory the excited UHR waves are trapped in striations. ${ }^{22}$ Their typical transverse sizes are much smaller than the transverse size of striations. ${ }^{23}$ Therefore the backscattered signal at the frequencies $f_{r} \pm f_{h}$ should be much stronger in the VHF and UHF ranges than in the HF range. Only recently, similar backscattered signals in the HF range were observed with the help of a special digital receiver. ${ }^{24}$ Note that up to now LH waves were not detected at oblique sounding.

Plasma perturbations caused by ionospheric modification by powerful $\mathrm{HF}$ radio waves can be investigated directly on board of a rocket crossing the heated region. Such an experiment was carried out by Kelley et $a l^{25}$ Transverse scales of striations, their elongation, and relative plasma depletion in- 
side them were found in this experiment. Later on the relative electron temperature enhancement inside striations was estimated $^{26}$ and also the excitation of drift waves was confirmed. $^{27}$

It is known from the theory that LH waves should be excited artificially in ionospheric modification experiments. ${ }^{28}$ Also, it is concluded from the analysis of the experimental data that LH waves are generated in the process of ionospheric heating. ${ }^{29}$ Indeed, the downshifted maximum (DM), one of the most prominent features of the secondary electromagnetic radiation, has an off-shift from the pump frequency equal to the lower hybrid resonance (LHR) frequency. Such off-shift appears if LH waves are involved in the decay process of a strong upper hybrid (UH) wave into another UH wave with smaller frequency and LH wave. ${ }^{29,30}$ While the processes with UH waves in ionospheric modification experiments are actively investigated, see, e.g., Refs. 23, 30, and 31 , the role of LH waves generated in such experiments and their parameters are still not clear enough. For example, we do not know typical amplitudes of LH waves excited in ionospheric modification experiments and their transverse sizes. That is why to detect LH waves directly by rocket or by backscattering from the Earth would be of prime importance. At the same time the possibility of such detection should be investigated theoretically. Recently the authors have published a paper discussing theoretically the mechanism of LH waves trapping associated with the waves transformation in elongated plasma depletions in the $2 \mathrm{D}$ case. ${ }^{32}$ It was shown that the trapping of LH waves in plasma depletions can take place not only for the frequencies below the LHR frequency of the background plasma (as was discussed theoretically before ${ }^{33,34}$ ) but also above it.

In the present paper the general theory of LH waves trapping in warm inhomogeneous plasma in the $3 \mathrm{D}$ case is given. The main attention is devoted to the trapping and excitation of LH waves in small-scale striations generated artificially in ionospheric modification experiments. Nevertheless, for completeness we consider also the trapping of LH in natural plasma depletions in the auroral upper ionosphere. It is shown that the results obtained in Refs. 33 and 34 for the explanation of LH waves "spikelets" are contained in our theory as a particular case. In relation with the planned ionospheric experiment (see Sec. V) typical intensities and the sizes of LH waves trapped in striations excited in ionospheric modification experiments are evaluated. It allows us to estimate the possibility of LH waves detection by backscattering from striations at VHF and UHF frequencies. Also the equations describing mutual transformation of electrostatic LH waves and electromagnetic whistler mode waves in a warm inhomogeneous plasma are derived. This part could be useful for the analysis of experimental data obtained in space and laboratory.

\section{BASIC EQUATIONS}

The procedure of obtaining the linear equation describing LH waves in a homogeneous plasma is well known, see, e.g., Ref. 35. If we take into account thermal corrections, for pure electrostatic perturbations in a homogeneous plasma an equation of the fourth order is easily obtained. In an inhomogeneous plasma the situation is more complicated. Let us assume that the magnetic field is directed along the $z$-axis, the inhomogeneity of plasma concentration exists along the $x$-axis, and along the $y$-axis the plasma is homogeneous. The corresponding equation describing such LH perturbations in the linear approximation was presented by the authors in Ref. 32. Here we are briefly reminded of the procedure of obtaining of such an equation. Let us introduce the electric potential for the $\mathrm{LH}$ perturbations in the form

$$
\Phi=\Phi_{0}(x) \exp \left[i\left(\omega t-k_{y} y-k_{z} z\right)\right]
$$

The potential $\Phi$ is connected with the electric field $\mathbf{E}$ $=-\nabla \Phi, \omega$ is the LH frequency, $k_{y}, k_{z}$ are the wave numbers along the $y$ and the $z$-axes correspondingly. From the equation of motion and the continuity equation for electrons we find their perturbation of concentration $n_{e}$. The perturbation of ion concentration $n_{i}$ is obtained from the Boltzmann equation. The link between the electric potential $\Phi_{0}$ and the perturbations $n_{i}$ and $n_{e}$ is given by the Poisson equation. After some transformations we arrive at the following differential equation describing LH perturbations in a warm inhomogeneous plasma stretched along the magnetic field line:

$$
\begin{aligned}
& R^{2}\left(\frac{\partial^{2}}{\partial x^{2}}-k_{y}^{2}\right)\left(\frac{\partial}{\partial x} \frac{\omega_{P i}^{2}}{\omega^{2}} \frac{\partial}{\partial x}-\frac{\omega_{P i}^{2}}{\omega^{2}} k_{y}^{2}\right) \Phi_{0} \\
& \quad+\frac{\partial}{\partial x}\left(\frac{\omega_{P e}^{2}}{\omega_{H e}^{2}}+1-\frac{\omega_{P i}^{2}}{\omega^{2}}\right) \frac{\partial}{\partial x} \Phi_{0}-k_{y}^{2}\left(\frac{\omega_{P e}^{2}}{\omega_{H e}^{2}}+1-\frac{\omega_{P i}^{2}}{\omega^{2}}\right) \Phi_{0} \\
& +\frac{\omega_{P e}^{2}}{\omega^{2}}\left(k_{z}^{2}-\kappa_{n} k_{y} \frac{\omega}{\omega_{H e}}\right) \Phi_{0}=0 .
\end{aligned}
$$

Here $R \approx \rho_{e}$, where $\rho_{e}$ is the Larmor radius of electrons $\rho_{e}=V_{T e} / \omega_{H e}, V_{T e}$ and $\omega_{H e}$ are the thermal velocity and the Larmor frequency of electrons, $\omega_{P e}=\left(4 \pi e^{2} N_{a} / m\right)^{1 / 2}$, $\omega_{P i}=\left(4 \pi e^{2} N_{a} / M\right)^{1 / 2}$ are the plasma frequencies of electrons and ions, $m$ and $M$ are the masses of electron and ion, $\kappa_{n}$ characterizes the inhomogeneity of plasma along the $x$-axis $\kappa_{n}=\partial N_{a} / N_{a} \partial x, N_{a}$ is the ambient plasma concentration equal to the sum of the homogeneous background concentration $N_{0}$ and small negative perturbation $\delta n(x)$ presenting the plasma distribution in a depletion $N_{a}=N_{0}+\delta n(x)$. Note, that a more accurate expression for the coefficient takes the form $R=\rho_{e}\left(1+1.5 T_{i} / T_{e}\right)^{1 / 2}$, see, e.g., Ref. 12. In the F-region of the ionosphere illuminated by powerful radio waves the temperature of electrons $T_{e}$ exceeds several times the temperature of ions $T_{i}$. Due to this later on we neglect the difference between $R$ and $\rho_{e}$.

In the geometric optics approximation when the dependence on the $x$-coordinate takes the form

$$
\Phi_{0} \propto \exp \left[-i \int^{x} k_{x}\left(x_{1}\right) d x_{1}\right]
$$

after the substitution of it in Eq. (2) we arrive at the following equation: 
$\rho_{e}^{2}\left(k_{x}^{2}+k_{y}^{2}\right)^{2}+\left(k_{x}^{2}+k_{y}^{2}\right)\left(1-\frac{\omega^{2}}{\omega_{\text {LHR }}^{2}}\right)+\frac{M}{m}\left(k_{z}^{2}-\kappa_{n} k_{y} \frac{\omega}{\omega_{H e}}\right)=0$,

where $\omega_{\text {LHR }}$ is the LHR frequency

$$
\omega_{\mathrm{LHR}}=\frac{\omega_{P i} \omega_{H e}}{\left(\omega_{P e}^{2}+\omega_{H e}^{2}\right)^{1 / 2}} .
$$

Note, that the LHR frequency depends on plasma concentration. Hence it varies with the $x$ coordinate because plasma is assumed to be inhomogeneous in this direction due to the presence of striations. An equation similar to Eq. (3) can be derived if the plasma is cylindrically symmetric. In this case the inhomogeneity of the background plasma exists along the radius $r$ while it is homogeneous in the azimuthal direction $\varphi$.

Equation (3) determines the dispersion relation $\omega$ $=\omega(\mathbf{k})$ for LH electrostatic perturbations. Earlier solutions of such an equation describing the trapping of $\mathrm{LH}$ waves in the 2D case $\left(k_{y}=0\right)$ were analyzed by the authors. ${ }^{32}$ It was shown that the trapping takes place for LH waves with the frequencies in the vicinity of the LHR frequency of the background plasma (above and below the LHR frequency depending on the longitudinal wave number and the relative depth of a depletion). Now we would like to consider a more general 3D case. Equation (3) without the last term $\left(\propto \kappa_{n} k_{y}\right)$ presents the well-known dependence of the LH wave frequency on the transverse $k_{x}, k_{y}$ and the longitudinal $k_{z}$ wave numbers taking into account thermal corrections, see, e.g., Ref. 12. The last term in this equation corresponds to the drift motion of electrons in an inhomogeneous plasma.

The solutions of Eq. (3) are the following:

$$
k_{x}^{2}=\frac{A(x)}{2 \rho_{e}^{2}}-k_{y}^{2} \pm \sqrt{\frac{A(x)^{2}}{4 \rho_{e}^{4}}-\frac{B(x)}{\rho_{e}^{2}}},
$$

where

$$
A(x)=\frac{\omega^{2}}{\omega_{\mathrm{LHR}}^{2}(x)}-1, \quad B(x)=\frac{M}{m}\left(k_{z}^{2}-k_{y} \kappa_{n} \frac{\omega}{\omega_{H e}}\right) .
$$

Later on we shall be interested in the waves with the frequencies not far from the LHR frequency. Due to this the ratio $\omega / \omega_{H e}$ in the coefficient $B$ approximately can be substituted by $\omega / \omega_{H e} \approx \sqrt{m / M}$.

\section{TRAPPING OF LH WAVES IN PLASMA DEPLETIONS IN A COLD PLASMA}

Earlier it was shown by the authors ${ }^{32}$ that in the $2 \mathrm{D}$ case the trapping of $\mathrm{LH}$ waves takes place in striations for a restricted range of frequencies in the vicinity of the LHR frequency. The reflection of energy occurs at some point inside plasma depletion where the function under the square root in Eq. (5) goes through zero. In the 2D case it happens only if we take into account the dependence of the coefficient $A$ on the $x$-coordinate. But such dependence in ionospheric plasma depletions is often rather weak enough. Indeed, typical variation of the coefficient $A$ in striations is estimated as $\delta A$ $=\omega^{2} / \omega_{P i}^{2} \delta n(x) / N_{0} \sim 10^{-3}$. In the 3D case there is another fac- tor that influences the behavior of the function $F$ under the square root in Eq. (5). In an explicit form this function $F(x)$ can be presented as

$$
F(x)=\left[\frac{\omega^{2}}{\omega_{\mathrm{LHR}}^{2}(x)}-1\right]^{2}-4 \rho_{e}^{2}\left[\frac{M}{m} k_{z}^{2}-\sqrt{\frac{M}{m}} \kappa_{n}(x) k_{y}\right] .
$$

It is clear that the magnitude and the sign of the function $F(x)$ varies with $x$ due to the presence of the last (drift) term. The idea that the drift of electrons influences the LH waves trapping was introduced earlier in Refs. 33 and 34. Estimates show than in the case of artificial plasma depletions (striations) the contribution of this drift term can be larger than $\delta A \cdot A$ if the perturbations are small-scale enough along the $y$-axis (the $k_{y}$ wave number is large). In such a case, we are able to neglect the variations of the function $A$ along the $x$-axis and to take into account only the changes with $x$ of the drift term.

Let us consider different cases described by Eq. (5). Suppose first that the first term under the square root is larger than the second term $A^{2} \gg 4 \rho_{e}^{2}|B(x)|$. It means that the LH frequency of the excited wave is not too close to the LHR frequency of the background plasma. In such a case one of the solutions of Eq. (5) is the following:

$$
k_{x}^{2}=\frac{B(x)}{A}-k_{y}^{2}
$$

This solution corresponds to the dispersion relation in the cold plasma approximation (the Larmor radius of the electron does not enter this solution). The right-hand side of Eq. (8) is positive if coefficients $A$ and $B$ are negative or positive simultaneously and the ratio $B(x) / A$ is large enough $B / A$ $>k_{y}^{2}$. The coefficient $A$ is definitely negative if the LH wave frequency is less than the minimal LHR frequency. At the same time the coefficient $B(x)$ can be negative only due to the input of the drift term. This case was considered previously in Refs. 33 and 34 with the aim to explain the observed bursts of LH waves ("spikelets") in natural plasma depletions in the auroral upper ionosphere. The second case when coefficients $A$ and $B$ are positive was missed in the investigations. ${ }^{33,34}$ The coefficient $A$ is positive for the waves with frequencies exceeding the LHR frequency. The coefficient $B$ is definitely positive if $k_{y} \kappa_{n} \leqslant 0$. Hence the wave propagation in the cold plasma approximation exists both for the frequencies either below or above the LHR frequency of the ambient plasma.

For model Gaussian distribution of plasma in a plasma depletion

$$
\delta n=-n_{0} \exp \left(-x^{2} / a^{2}\right)
$$

the coefficient $\kappa_{n}$ is the following:

$$
\kappa_{n}=\frac{n_{0}}{N_{0}} \frac{2 x}{a^{2}} \exp \left(-\frac{x^{2}}{a^{2}}\right),
$$

where $a$ describes the transverse size of a striation. Later on we discuss the conditions for wave propagation and trapping assuming that $x>0$. This case corresponds also to the axial 
symmetric cavity if we substitute in the geometric optics approximation $x \rightarrow r$.

For the model distribution of plasma in a striation [Eq. (9)] we find the $k_{x}$ number in an explicit form,

$k_{x}^{2}=\frac{M}{m}\left[k_{z}^{2}-2 \sqrt{\frac{m}{M}} k_{y} \frac{n_{0}}{N_{0}} \frac{x}{a^{2}} \exp \left(-\frac{x^{2}}{a^{2}}\right)\right] \frac{\omega_{\mathrm{LHR}}^{2}}{\omega^{2}-\omega_{\mathrm{LHR}}^{2}}-k_{y}^{2}$.

Suppose that the LH wave frequency is less than the minimal LHR frequency (the coefficient $A$ is negative). It is clear that the term in square brackets in Eq. (10) is negative for $x>0$ only if the wave number $k_{y}$ is positive and the corresponding $k_{z}$ numbers are small enough $\left|k_{z}\right|$ $<(m / M)^{1 / 4}\left(n_{0} / N_{0}\right)^{1 / 2}\left(k_{y} / a\right)^{1 / 2}$. The reflection of waves takes place at the turning points $x_{1}, x_{2}$, where $k_{x}^{2}$ given by Eq. (10) becomes equal to zero. Similarly for the wave frequencies higher than the LHR frequency the trapping can take place only for negative $k_{y}$ numbers. The stationary states of the trapped LH waves exist for such frequencies, that

$$
\int_{x_{1}}^{x_{2}} \sqrt{\frac{B(x)}{A}-k_{y}^{2}} d x=\pi(n+1 / 2),
$$

where $n$ is an integer. Equation (11) determines a discrete set of eigenfrequencies for which the stationary states exist. It is clear that the corresponding $k_{x}$ numbers [see Eq. (10)] should not be too small and the integration region $\Delta x=x_{2}-x_{1}$ not too narrow. Indeed, Eq. (11) shows that the stationary states exist only if

$$
\int_{x_{1}}^{x_{2}} k_{x} d x \geqslant \pi / 2
$$

To find concrete values of the eigenfrequencies, let us discuss a simple analytical model with the Gaussian distribution of plasma in a striation [see Eq. (9)]. Suppose that the $k_{z}$ number in Eq. (10) is so small that it can be neglected. Also we neglect the variation of the LHR frequency in the coefficient $A$, assuming that $\omega_{\mathrm{LHR}}=\omega_{\mathrm{LHR}}^{(0)}=$ const. In this case we arrive at the following equation describing the trapped eigenstates in the cold plasma approximation:

$$
\begin{aligned}
& \int_{\xi_{1, n}}^{\xi_{2, n}} \sqrt{\xi \exp \left(-\xi^{2}\right)-\epsilon_{n}} d \xi \\
& \quad=\pi(n+1 / 2)\left(\frac{m}{M}\right)^{1 / 4}\left(\frac{N_{0}}{n_{0}}\right)^{1 / 2} \sqrt{\frac{\omega_{\mathrm{LHR}}^{(0)}-\omega_{n}}{a k_{y} \omega_{\mathrm{LHR}}^{(0)}}} .
\end{aligned}
$$

Here,

$$
\xi=x / a, \quad \epsilon_{n}=k_{y} a\left(\frac{m}{M}\right)^{1 / 2} \frac{N_{0}}{n_{0}} \frac{\omega_{\mathrm{LHR}}^{(0)}-\omega_{n}}{\omega_{\mathrm{LHR}}^{(0)}} .
$$

For the first lowest trapped modes $n=0,1,2, \ldots$ and $\left|k_{y}\right| a$ $\gg 1$ Eq. (12) is solved analytically. Indeed, near its maximum the potential $U(\xi)=\xi \exp \left(-\xi^{2}\right)$ can be presented as

$$
U(\xi) \approx U_{m}+\frac{1}{2} \frac{d^{2} U}{d \xi^{2}}\left(\xi-\xi_{m}\right)^{2},
$$

where $\xi_{m}=1 / \sqrt{2}, U_{m}=U\left(\xi_{m}\right)$. After the substitution of Eq. (13) into Eq. (12) and integration with respect to $\xi$ we find the set of eigenfrequencies $\omega_{n},\left(n=0,1, \ldots, n_{\max }\right)$ in an explicit form

$$
\begin{aligned}
\omega_{n}= & \omega_{\mathrm{LHR}}^{(0)}\left\{1-\frac{1}{k_{y} a} \sqrt{\frac{M}{m}} \frac{n_{0}}{N_{0}}\right. \\
& \left.\times\left[U_{m}-\frac{2 n+1}{\left|k_{y}\right| a} \sqrt{U_{m}} \exp (-1 / 4)\right]\right\},
\end{aligned}
$$

where $2 n_{\max } \leqslant \sqrt{U_{m}} \exp (1 / 4)\left|k_{y}\right| a-1$. It is seen from Eq. (14) that the eigenfrequencies can be smaller or exceed the LHR frequency depending on the sign of $k_{y}$. Also it is clear that the trapped modes with the frequencies below and above the LHR frequency of the background plasma are localized mainly in the regions with the largest gradients of plasma concentration. The modes with different signs of the $k_{y}$ number rotate in opposite directions. ${ }^{33,34}$

Note, that the maximum of the function $U(\xi)$ $=\xi \exp \left(-\xi^{2}\right)$ under the square root in the left-hand side of Eq. (12) is $U_{m} \approx 0.7$. According to Eq. (12) the eigenvalues $\epsilon_{n}$ should be always smaller than this value, hence,

$$
\frac{\left|\omega_{\mathrm{LHR}}^{(0)}-\omega_{n}\right|}{\omega_{\mathrm{LHR}}^{(0)}} \leqslant 0.7\left(\frac{M}{m}\right)^{1 / 2} \frac{n_{0}}{N_{0}} \frac{1}{\left|k_{y}\right| a} .
$$

At the same time the eigenfrequencies $\omega_{n}$ should not be too close to the LHR frequency $A^{2} \gg 2 \rho_{e}^{2}|B|$. Otherwise, according to Eq. (5), the cold plasma approximation is not valid and thermal effects (finite magnitude of the electron Larmor radius) should be taken into account. Due to the last restriction we avoid singularities and discontinuities in the $k_{x}$ numbers that appear in Eq. (10) if $\omega \rightarrow \omega_{\mathrm{LHR}}$. An estimate for the validity of the cold plasma approximation can be presented in the explicit form

$$
\frac{\left|\omega_{\mathrm{LHR}}^{(0)}-\omega_{n}\right|}{\omega_{\mathrm{LHR}}^{(0)}} \gg \rho_{e} \sqrt{\frac{\left|k_{y}\right|}{a}}\left(\frac{M}{m}\right)^{1 / 4}\left(\frac{n_{0}}{N_{0}}\right)^{1 / 2} .
$$

It is quite clear that the conditions (15) and (16) should not contradict to each other. It implies the following restriction on the $k_{y}$ numbers:

$$
\left|k_{y}\right|^{3} \rho_{e}^{2} a \ll\left(\frac{M}{m}\right)^{1 / 2} \frac{n_{0}}{N_{0}} .
$$

\section{TRAPPING OF LH WAVES IN PLASMA DEPLETIONS IN A WARM PLASMA}

Now let us discuss the mechanism of trapping in a warm plasma. As it was done previously by the authors in the $2 \mathrm{D}$ case, ${ }^{32}$ we find the ratio of the group and the phase velocities of the LH wave along the $x$-axis in the 3D case, 


$$
\frac{V_{g}}{V_{p h}} \approx 2 \frac{\omega_{\mathrm{LHR}}^{(0) 2}}{\omega^{2}} k_{x}^{2} \rho_{e}^{2}\left[1-\frac{M}{m} \frac{k_{z}^{2}-\kappa_{n} k_{y} \sqrt{\frac{m}{M}}}{\left(k_{x}^{2}+k_{y}^{2}\right)^{2} \rho_{e}^{2}}\right] .
$$

This relation in the case when $k_{z}^{2}-\kappa_{n} k_{y} \sqrt{m / M}>0$ determines a particular $k_{x}^{(*)}$ number for which the group velocity becomes equal to zero

$$
k_{x}^{(*) 2}=\frac{1}{\rho_{e}} \sqrt{\frac{M}{m}}\left(k_{z}^{2}-\kappa_{n} k_{y} \sqrt{\frac{m}{M}}\right)^{1 / 2}-k_{y}^{2} .
$$

Assume that the frequency of the LH wave $\omega$ and the wave numbers $k_{y}$ and $k_{z}$ are such that the following conditions are fulfilled:

$\omega^{2}-\omega_{\mathrm{LHR}}^{(0) 2}>2 \rho_{e}^{2} k_{y}^{2} \omega_{\mathrm{LHR}}^{(0) 2}, \quad \omega^{2}-\omega_{\mathrm{LHR}}^{(0) 2}>2 \rho_{e}\left|k_{z}\right| \sqrt{\frac{M}{m}} \omega_{\mathrm{LHR}}^{(0) 2}$.

It means that the frequency $\omega$ should be larger than the LHR frequency. In the discussed case, according to Eq. (5), two types of propagating waves that correspond to plus and minus signs before the root exist. Let the $k_{y}$ number be negative. As the coefficient $B(x)$ grows for $x$ increasing from $x=0$, at some particular point $x_{1}$ the following relation takes place (if the inhomogeneity is not too weak):

$$
\kappa_{n}\left(x_{1}\right)=\sqrt{\frac{m}{M}} \frac{1}{4 \rho_{e}^{2} k_{y}}\left[\frac{M}{m} 4 \rho_{e}^{2} k_{z}^{2}-A^{2}\right] .
$$

It is easy to prove that at this point the group velocity becomes equal to zero and at the same time two types of waves determined by Eq. (5) have equal $k_{x}$ wave numbers. According to the analysis presented earlier ${ }^{32}$ the transformation of waves (with plus and minus signs) takes place at this point $x=x_{1}$. But in contrast with the 2D case discussed in Ref. 32, the transformation is not total. For larger $x$ values (closer to the boundary of depletion) in the general case there is another point $x_{2}$ at which the same relation as Eq. (19) takes place. So the region $x_{1}<x<x_{2}$ is not transparent for the $\mathrm{LH}$ waves. In the region where $x>x_{2}$ the propagation of the $\mathrm{LH}$ wave once again is possible. If the leakage of energy through the mentioned region $x_{1}<x<x_{2}$ is small enough, we may argue that the LH wave is trapped in the inner region $x<x_{1}$. The leakage can be estimated by the coefficient of barrier transparency $P$,

$$
\begin{aligned}
P \approx & \exp \left[-\sqrt{\frac{2}{A-2 \rho_{e}^{2} k_{y}^{2}}\left(\frac{M}{m}\right)^{1 / 4}\left|k_{y}\right|^{1 / 2}}\right. \\
& \times \int_{x_{1}}^{x_{2}} \sqrt{\kappa_{n}(x)-\sqrt{\frac{m}{M}} \frac{A^{2}-4 \frac{M}{m} k_{z}^{2} \rho_{e}^{2}}{4 \rho_{e}^{2}\left|k_{y}\right|} d x} .
\end{aligned}
$$

It is possible to consider also one more case
$\omega^{2}-\omega_{\mathrm{LHR}}^{(0) 2}>2 \rho_{e}^{2} k_{y}^{2} \omega_{\mathrm{LHR}}^{(0) 2}, \quad \omega^{2}-\omega_{\mathrm{LHR}}^{(0) 2}<2 \rho_{e} k_{z} \sqrt{\frac{M}{m}} \omega_{\mathrm{LHR}}^{(0) 2}$.

Now the trapping can exist only in some intermediate region inside the plasma depletion $x_{1}<x<x_{2}$ for positive values of $k_{y}$. Indeed the turning points are determined similarly to Eq. (21). Near the center of depletion $x<x_{1}$ and at large enough distances $x>x_{2}$, there is no propagation because the wave is attenuated. Note, that the two cases considered above are distinguished by the direction of phase velocity along the $y$-axis (due to positive and negative signs of the $k_{y}$ wave numbers).

For the frequencies slightly below the LHR frequency of the ambient plasma, thermal effects can also influence the process of trapping in striations. Such a case was considered previously by the authors in the $2 \mathrm{D}$ approximation, taking into account the variation of the LHR frequency along the $x$-axis $\omega_{\text {LHR }}(x) .^{32}$ Similar to the analysis presented above, in this case the mutual transformation of different waves takes place at the points where the group velocity is equal to zero. If we neglect the dependence of $\omega_{\text {LHR }}$ on the $x$-coordinate, it can be shown that no mutual transformation of waves exists for the frequencies $\omega \leqslant \omega_{\mathrm{LHR}}$. At the same time, according to Eq. (5) the propagation of LH waves is still possible in the regions where $B(x)<0$.

So, the LH waves trapping in plasma depletions exists for the frequencies below the LHR frequency of the background plasma and above it. As it was shown above, to correctly analyze the trapping in the vicinity of the LHR frequency, thermal effects should be taken into account. In this case the trapping is associated with the transformation of waves.

\section{EXCITATION OF LH WAVES IN STRIATIONS}

Usually it is supposed that LH waves in heating experiments are excited due to the decay of a strong UHR wave (with a pump frequency) trapped in a striation into another UHR wave with smaller frequency and the LH wave leaking from a striation. ${ }^{30}$ In such a case, LH oscillations with rather broad frequency spectrum are generated. Another possibility can be realized when LH waves are excited by two strong UHR oscillations trapped in a striation. These UHR oscillations with different frequencies $\omega_{1}$ and $\omega_{2}$ are generated by simultaneous action of two powerful ordinary HF radio waves with the frequencies $\omega_{1}$ and $\omega_{2}$. The frequency of the excited LH wave is equal to the difference of frequencies $\omega_{1}-\omega_{2}$. In such an experiment LH waves with rather high amplitude are generated at a given frequency $\omega_{3}=\omega_{1}-\omega_{2}$. This experiment can be carried out with the help of the existing heating facilities.

The aim of this section is to theoretically discuss the above-mentioned planned experiment and to estimate the typical amplitude of the LH wave and its transverse scale. We assume that the LH wave is excited with a frequency above the LHR frequency and this wave is also trapped in a striation. Let us designate by the indices $j=1,2$ strong UHR oscillations and by the index $j=3$ the LH oscillations. For 
simplicity we shall consider the excitation of LH waves trapped inside a given striation in the $2 \mathrm{D}$ case.

First of all, let us prove that different LH eigenmodes $\partial \Phi_{3, n} / \partial x$ that correspond to different eigenvalues $\omega_{n}$ are orthogonal. An approximate equation for the potential $\Phi_{3}$ follows from Eq. (2) with $k_{y}=0$,

$$
\rho_{e}^{2} \frac{\partial^{4} \Phi_{3}}{\partial x^{4}}+\frac{\partial}{\partial x}\left(\frac{\omega^{2}}{\omega_{\text {LHR }}^{2}}-1\right) \frac{\partial \Phi_{3}}{\partial x}+\frac{M}{m} k_{z}^{2} \Phi_{3}=0 .
$$

The details of obtaining such an equation can be found in Ref. 32. Let us multiply Eq. (24) for the function $\Phi_{3, n}$ by $\Phi_{3, m}$ and the similar equation for $\Phi_{3, m}$ by $\Phi_{3, n}$. After that we subtract the second obtained equation from the first one and the difference integrate with respect to $x$. As a result we arrive at the relation

$$
\left(\omega_{3, n}-\omega_{3, m}\right) \int \frac{\partial \Phi_{3, n}}{\partial x} \frac{\omega_{3}}{\omega_{\mathrm{LHR}}^{2}(x)} \frac{\partial \Phi_{3, m}}{\partial x} d x=0 .
$$

It is seen from Eq. (25) that eigenfunctions $\partial \Phi_{3, n} / \partial x$ with different indices $m \neq n$ are indeed orthogonal with the weight $\omega_{0}^{2} / \omega_{\text {LHR }}^{2}$.

To discuss the excitation of the LH wave trapped in a striation we use the equation of motion for electrons with the nonlinear term $(\mathbf{v} \nabla) \mathbf{v}$,

$$
\begin{aligned}
\frac{\partial \mathbf{v}_{\mathbf{e}}^{(3)}}{\partial t}= & \frac{e}{m} \nabla \Phi_{3}-\omega_{H e}\left[\mathbf{v}_{\mathbf{e}}^{(3)} \times \mathbf{H}\right]-\frac{T_{e}}{m N_{a}} \nabla n_{e}^{(3)} \\
& -\left[\mathbf{v}_{\mathbf{e}}^{(\mathbf{1})} \nabla\right] \mathbf{v}_{\mathbf{e}}^{(\mathbf{2})}-\left[\mathbf{v}_{\mathbf{e}}^{(\mathbf{2})} \nabla\right] \mathbf{v}_{\mathbf{e}}^{(\mathbf{1})} .
\end{aligned}
$$

Here we present the electric fields that correspond to UHR waves in the following form:

$$
\begin{aligned}
& E_{1, x}=-\frac{1}{2} \frac{\partial \Phi_{1}}{\partial x}(x) \exp \left[i \omega_{1} t-\int^{z} k_{z}^{(1)}\left(z_{1}\right) d z_{1}\right]+\text { c.c. } \\
& E_{2, x}=-\frac{1}{2} \frac{\partial \Phi_{2}}{\partial x}(x) \exp \left[i \omega_{2} t-\int^{z} k_{z}^{(2)}\left(z_{1}\right) d z_{1}\right]+\text { c.c. }
\end{aligned}
$$

where $\Phi_{1}$ and $\Phi_{2}$ are the potentials of the trapped UHR waves, $\omega_{1}$ and $\omega_{2}$ are their frequencies, c.c. means complex conjugate, $\omega_{3}=\omega_{1}-\omega_{2}, n_{e}^{(3)}, \Phi_{3}$ are the frequency of the $\mathrm{LH}$ wave, perturbation of plasma concentration, and the electric potential related to this wave.

The continuity equation for the $\mathrm{LH}$ wave also contains a nonlinear term

$$
\frac{\partial}{\partial t} n_{e}^{(3)}+\nabla N_{0} \mathbf{v}_{\mathbf{e}}^{(3)}+\nabla\left[n_{e}^{(1)} \mathbf{v}_{\mathbf{e}}^{(2)}\right]+\nabla\left[n_{e}^{(2)} \mathbf{v}_{\mathbf{e}}^{(1)}\right]=0 .
$$

The velocities $\mathbf{v}_{\mathbf{e}}^{(1)}, \mathbf{v}_{\mathbf{e}}^{(2)}$ and the corresponding concentrations $n_{e}^{(1)}, n_{e}^{(2)}$ are determined from the linear equations for electrons neglected for simplicity of the influence of the magnetic field. Indeed, in the F-region of the ionosphere usually the pump frequencies $\omega_{1}, \omega_{2}$ are several times larger than the Larmor frequency of the electron $\omega_{H e}$. After some transformations we arrive at the following approximate equation for the excited LH waves:

$$
\begin{aligned}
\rho_{e}^{2} \frac{\partial^{4} \Phi_{3}}{\partial x^{4}}+ & \frac{\partial}{\partial x}\left(\frac{\omega_{3}^{2}}{\omega_{\mathrm{LHR}}^{2}}-1\right) \frac{\partial \Phi_{3}}{\partial x}+\frac{M}{m} k_{z}^{(3) 2} \Phi_{3} \\
= & \frac{e}{4 m \omega_{1} \omega_{2}} \frac{\partial^{2}}{\partial x^{2}}\left(\frac{\partial \Phi_{1}}{\partial x} \frac{\partial \Phi_{2}^{*}}{\partial x}\right) \\
& \times \exp \left(-i\left\{\int^{z}\left[k_{z}^{(1)}\left(z_{1}\right)-k_{z}^{(2)}\left(z_{1}\right)\right] d z_{1}-k_{z}^{(3)} z\right\}\right) .
\end{aligned}
$$

A similar equation was derived previously in a homogeneous plasma with the aim to discuss the decay of a strong UHR wave into another UHR wave with smaller frequency and the LH wave. ${ }^{28}$ Our Eq. (29) is more general. It is valid also if all three interacting waves are trapped inside striation. It reduces to the equation obtained earlier in case of a homogeneous plasma. ${ }^{28}$ Below we apply Eq. (29) to investigate the excitation of LH wave by the action of two strong UHR waves $\Phi_{1}$ and $\Phi_{2}$ with different frequencies $\omega_{1}$ and $\omega_{2}$.

Note, that the $k_{z}$ wave numbers for UHR eigenmodes trapped inside striations according to Ref. 23 change rather quickly with $z$. That is why we presented the $z$-dependence for them in Eq. (27) in the form of integrals. At the same time the LH oscillations have very weak dependence on $z$. Indeed, the corresponding vertical scale for LH oscillations is much larger than for the UHR oscillations. So, in the present paper we neglect vertical inhomogeneity while describing the LH waves. Due to the changes along the $z$-axis of the $k_{z}$ wave numbers, the matching conditions along this axis $k_{z}^{(1)}(z)=k_{z}^{(2)}(z)+k_{z}^{(3)}$ are fulfilled only within a finite range of heights, hence restricting the efficiency of the waves transformation.

Taking into account that the functions $\partial \Phi_{3, n} / \partial x$ which correspond to different trapped eigenstates are orthogonal [see Eq. (25)], it is reasonable to obtain an equation for such functions. If we differentiate both sides of Eq. (29) with respect to $x$, the solution of the obtained equation can be represented as a combination of orthogonal functions

$$
\frac{\partial \Phi_{3}}{\partial x}=\sum_{n} c_{n}(z) \frac{\partial \Phi_{3, n}}{\partial x},
$$

where $\partial \Phi_{3, n} / \partial x$ are the eigenfunctions with the eigenfrequencies of the trapped LH modes $\omega_{3, n}, c_{n}(z)$ are the coefficients describing the excitation of different modes. We suppose that the functions $\partial \Phi_{3, n} / \partial x$ are normalized. Usually it is accepted that the norm is equal to unity $\int\left(\partial \Phi_{3, n} / \partial x\right)^{2} \omega_{3}^{2} / \omega_{\mathrm{LHR}}^{2} d x=1$.

To investigate the excitation of different trapped eigenmodes of LH we use Eqs. (29) and (30). In deriving the corresponding equation we take into account that $\Phi_{3, n}$ are the solutions of the homogeneous Eq. (24) with the eigenfrequencies $\omega_{3, n}$. As a result we arrive at the equation 


$$
\begin{aligned}
-2 i \frac{M}{m} k_{z}^{(3)} \sum_{n} \frac{\partial c_{n}}{\partial z} \frac{\partial \Phi_{3, n}}{\partial x}+\sum_{n} c_{n} \frac{\partial^{2}}{\partial x^{2}}\left(\frac{\omega_{3, n}^{2}-\omega_{3}^{2}}{\omega_{\mathrm{LHR}}^{2}}\right) \frac{\partial \Phi_{3, n}}{\partial x} \\
=\frac{e}{4 m \omega_{1} \omega_{2}} \frac{\partial \Phi_{3 n}}{\partial x} \frac{\partial^{3}}{\partial x^{3}}\left(\frac{\partial \Phi_{1}}{\partial x} \frac{\partial \Phi_{2}^{*}}{\partial x}\right) \\
\quad \times \exp \left(-i\left\{\int^{z}\left[k_{z}^{(1)}\left(z_{1}\right)-k_{z}^{(2)}\left(z_{1}\right)\right] d z_{1}-k_{z}^{(3)} z\right\}\right) .
\end{aligned}
$$

Equation (31) is valid if the absolute value of the derivative $\left|\partial c_{n} / \partial z\right|$ is rather small $\left|\partial c_{n} / \partial z\right| \ll k_{z}^{(3)}\left|c_{n}\right|$. The most efficient excitation is expected for one particular mode $c_{r}$ for which there is a resonance between the frequencies of interacting waves with the eigenfrequency of this mode $\omega_{1}-\omega_{2}=\omega_{3, r}$. To find the relation describing the excitation of this particular mode, first, we multiply both sizes of Eq. (31) by $\omega_{3}^{2} / \omega_{\mathrm{LHR}}^{2} \partial \Phi_{3, r} / \partial x$, integrate with respect to $x$ and after that, integrate the obtained equation with respect to $z$. Neglecting for simplicity the interaction of different LH modes described by the second term in Eq. (31) we arrive at the relation

$$
\begin{aligned}
\max \left|c_{r}\right|= & \frac{e}{8 M k_{z}^{(3)} \omega_{1} \omega_{2}}\left|\int_{-\infty}^{\infty} \exp \left(i\left\{\int^{z}\left[k_{z}^{(1)}\left(z_{1}\right)-k_{z}^{(2)}\left(z_{1}\right)\right] d z_{1}-k_{z}^{(3)}\right\}\right) d z\right| \\
& \times\left|\int \frac{\partial \Phi_{3, r}}{\partial x} \frac{\omega_{3}^{2}}{\omega_{\mathrm{LHR}}^{2}} \frac{\partial^{3}}{\partial x^{3}}\left(\frac{\partial \Phi_{1}}{\partial x} \frac{\partial \Phi_{2}^{*}}{\partial x}\right) d x\right|
\end{aligned}
$$

From this relation it is seen that the efficiency of excitation, given by the coefficient $c_{r}$, is determined by the distribution of the UH and LH oscillations in a cross section of a striation (the functions $\partial \Phi_{j} / \partial x$ ) and also by the matching conditions between the longitudinal wave numbers $k_{z}^{(j)}$ of the interacting waves. Note, that due to the trapping all three functions $\partial \Phi_{j} / \partial x$ are wave packets localized inside a given striation. The most efficient interaction between the waves takes place if the transverse scale of oscillations of $\mathrm{LH}$ wave is close to the characteristic size determined by the product $\partial \Phi_{1} / \partial x \partial \Phi_{2}^{*} / \partial x$

To estimate the magnitude of the coefficient $c_{r}$ we discuss the case when the profile of a striation is given by Eq. (9). It is convenient to introduce a new dimensionless variable $\zeta=\mu x$, where

$$
\mu=\left(\frac{n_{0}}{N_{0}}\right)^{1 / 4}\left(\frac{\bar{\omega}}{\sqrt{\gamma_{\perp}} V_{T e} a}\right)^{1 / 2}
$$

is a characteristic wave number, $\bar{\omega}=\omega_{P e}\left(N_{0}\right), \gamma_{\perp}=3$ is the adiabatic coefficient. Near the center the Gaussian profile (9) can be substituted by a parabolic one $\exp \left(-x^{2} / a^{2}\right)$ $\approx 1-x^{2} / a^{2}$. It is well known that the eigenfunctions for such a profile are determined by Hermite polynomials.

To estimate the amplitude of the LH oscillations let us take the distributions of two UHR waves that correspond to the fourth and the second eigenmodes in a given striation

$$
\begin{aligned}
& \frac{\partial \Phi_{1}}{\partial \zeta}=C_{1} \frac{1}{(384 \sqrt{\pi})^{1 / 2}} \exp \left(-\frac{\zeta^{2}}{2}\right) H_{4}(\zeta), \\
& \frac{\partial \Phi_{2}}{\partial \zeta}=C_{2} \frac{1}{(8 \sqrt{\pi})^{1 / 2}} \exp \left(-\frac{\zeta^{2}}{2}\right) H_{2}(\zeta) .
\end{aligned}
$$

Here $H_{4}(\zeta)$ and $H_{2}(\zeta)$ are the Hermite polynomials of the fourth and the second order correspondingly, $C_{1}$ and $C_{2}$ are the amplitudes of the UHR waves. Note that eigenfunctions describing the distribution of UHR waves $\partial \Phi_{1} / \partial \zeta$ and $\partial \Phi_{2} / \partial \zeta$ are normalized in such a way that

$$
\int\left(\frac{\partial \Phi_{1,2}}{\partial \zeta}\right)^{2} d \zeta=C_{1,2}^{2}
$$

According to Eqs. (34) and (35) and Eq. (36) in Cartesian coordinates $\{x, y, z\}$ the electric fields of the UHR waves are given by

$$
\begin{aligned}
& \frac{\partial \Phi_{1}}{\partial x}=\frac{C_{1} \mu}{(384 \sqrt{\pi})^{1 / 2}} \exp \left[-\frac{(\mu x)^{2}}{2}\right] H_{4}(\mu x), \\
& \frac{\partial \Phi_{2}}{\partial x}=\frac{C_{2} \mu}{(8 \sqrt{\pi})^{1 / 2}} \exp \left[-\frac{(\mu x)^{2}}{2}\right] H_{2}(\mu x) .
\end{aligned}
$$

Now we need to specify the distribution of the LH wave in a striation. For rather crude estimates we assume that the trapped LH mode oscillates in some inner part of a striation with the $k_{x}$ number $k_{x} \approx \sqrt{A / 2 \rho_{e}^{2}}$; see Eq. (5), where $A$ $\approx 2 \rho_{e} \sqrt{B}$. As a result we find the model distribution of the LHR oscillations in a striation

$$
\frac{\partial \Phi_{3, r}}{\partial \zeta}=\frac{\sqrt{2}}{\pi^{1 / 4} \sqrt{\mu \Delta \zeta}} \sin \left(k_{\zeta} \zeta\right) \exp \left[-\left(\frac{\zeta}{\Delta \zeta}\right)^{2}\right] .
$$

Here $k_{\zeta}$ is a dimensionless transverse wave number for the trapped LH mode, given by

$$
k_{\zeta}=\left(\sqrt{\frac{M}{m}} \frac{k_{z}}{\rho_{e}}\right)^{1 / 2} \frac{\left(\sqrt{\gamma_{\perp}} a V_{T e}\right)^{1 / 2}}{\bar{\omega}^{1 / 2}}\left(\frac{N_{0}}{n_{0}}\right)^{1 / 4},
$$

$\Delta \zeta$ is a dimensionless size of the region where the LH mode is localized inside a given striation [determined by the turning points $x_{0}^{(1)}$ and $\left.x_{0}^{(2)}\right]$. Taking into account that the ratio $\omega_{3}^{2} / \omega_{\mathrm{LHR}}^{2}$ is close to unity, we assume that the function 
$\partial \Phi_{3, r} / \partial \zeta$ is normalized in such a way that $\int\left(\partial \Phi_{3, r} / \partial x\right)^{2} d x$ $=1$. Now we need to substitute the introduced above distributions of the UHR and LH waves into Eq. (32). The contribution to $c_{r}$ from the transverse distribution is given by the integral

$$
\int \frac{\partial \Phi_{3, r}}{\partial x} \frac{\partial^{3}}{\partial x^{3}}\left(\frac{\partial \Phi_{1}}{\partial x} \frac{\partial \Phi_{2}^{*}}{\partial x}\right) d x=\mu^{5} \int \frac{\partial \Phi_{3, r}}{\partial \zeta} \frac{\partial^{3}}{\partial \zeta^{3}}\left(\frac{\partial \Phi_{1}}{\partial \zeta} \frac{\partial \Phi_{2}^{*}}{\partial \zeta}\right) d \zeta .
$$

It is seen from Eq. (40) that the efficiency of the waves transformation increases rapidly $\left(\propto \mu^{5}\right)$ for small-scale waves. For numerical estimates we take $k_{z} \approx 4 \times 10^{-4} \mathrm{~cm}^{-1}$, $\rho_{e}=1.5 \mathrm{~cm}, n_{0} / N_{0}=0.06, a=5 \times 10^{2} \mathrm{~cm}, \Delta \zeta=1.6$. The corresponding wave number $k_{\zeta}$ is equal to $k_{\zeta} \approx 5.4$.

After numerical integration with respect to $\zeta$ in the righthand side of Eq. (40) we find

$$
\int \frac{\partial \Phi_{3, r}}{\partial x} \frac{\partial^{3}}{\partial x^{3}}\left(\frac{\partial \Phi_{1}}{\partial x} \frac{\partial \Phi_{2}^{*}}{\partial x}\right) d x \approx-20 \mu^{9 / 2} C_{1} C_{2} .
$$

As a result the amplitude of the excited LH wave takes the form

$$
\max \left|c_{r}\right| \approx \frac{e \Delta z}{M k_{z}^{(3)} \omega_{1} \omega_{2}} 2.5 \mu^{9 / 2} C_{1} C_{2},
$$

where $\Delta z$ describes the distance along the $z$-axis within which the matching conditions for the interacting waves are fulfilled

$$
\left|\int_{0}^{\Delta z}\left[k_{z}^{(1)}\left(z_{1}\right)-k_{z}^{(2)}\left(z_{1}\right)\right] d z_{1}-k_{z}^{(3)} \Delta z\right| \leqslant \frac{\pi}{2} .
$$

The length $\Delta z$ can be estimated based on the results obtained in Ref. 23. Assuming that $k_{z}^{(1)} \sim k_{z}^{(2)} \sim k_{z}^{(3)}$ we find

$$
\Delta z \approx \frac{\omega_{H e}}{\omega_{P e}\left(N_{0}\right)} \frac{\left[L_{z} k_{z}^{(3)}\right]^{1 / 2}}{\mu},
$$

where $L_{z}$ is the scale of inhomogeneity along the $z$-axis. The distance $\Delta z$ for typical conditions of the HF heating has the order of magnitude $\Delta z \sim 10^{3} \mathrm{~cm}$.

According to Eq. (42) the amplitude of the LH wave is sensitive to the magnitude of the characteristic wave number $\mu$, which in turn depends on parameters of a striation (relative depth $n_{0} / N_{0}$ and the half-width $a$ ). Also the LH wave amplitude quickly grows with the increase of the UHR wave amplitudes $C_{1}$ and $C_{2}$. Unfortunately, estimates based on different theoretical approaches give quite different values for UHR wave amplitudes that deviate approximately two orders of magnitude. ${ }^{23,31,36}$ Due to this it is rather difficult to obtain reliable estimates for typical amplitudes of the LH waves excited in heating experiments. We shall take some intermediate values $E_{1} \sim E_{2} \sim 5 \mathrm{~V} / \mathrm{m}$, that are close to estimates obtained in Ref. 23. In this case we find an estimate of $E_{3}$ $\approx(0.3-0.5) \mathrm{V} / \mathrm{m}$ for the amplitude of the excited LH wave trapped inside striation. The corresponding relative perturbations of plasma concentration with the LHR frequency due to such a field achieves the value $n^{(3)} / N_{0} \sim(0.01-0.02)$ and the typical transverse wave numbers are $k_{x} \approx 0.2-0.02 \mathrm{~cm}^{-1}$. Probably such perturbations can be detected with the help of
VHF and UHF radars by backscattering (oblique sounding) at displaced frequencies $f=f_{r} \pm f_{\mathrm{LH}}$, where $f_{r}$ is the radar frequency, $f_{\mathrm{LH}}$ is the frequency of the excited LH wave.

\section{TRANSFORMATION OF ELECTROSTATIC AND ELECTROMAGNETIC WAVES IN THE LH FREQUENCY RANGE}

Interaction between electrostatic and electromagnetic waves in the LH frequency range attracts significant attention due to existing experimental results in space ${ }^{1-4}$ and in laboratory plasmas. ${ }^{14,15}$ Several theoretical papers were presented to discuss the transformation of whistler mode waves into electrostatic LH waves in a given plasma depletion with the application to natural plasma processes in the ionosphere. $^{37,38}$

In this section we would like to analyze an opposite case, the excitation of electromagnetic fields localized in space LH electrostatic oscillations. The possibility to generate whistler mode waves due to the scattering on striations of propagating LH electrostatic oscillations was analyzed previously in the stochastic approach. ${ }^{39}$ Now we consider the excitation of a whistler mode wave by a localized wave packet of LH waves trapped in a given striation. In previous sections it was assumed that the electric field of the excited LH waves is purely electrostatic. At the same time in real conditions the electric field of the $\mathrm{LH}$ wave with a finite wave number has an admixture of the solenoidal field. Also, in the LH frequency range besides electrostatic there exist electromagnetic whistler mode waves. These two types of waves can have the same frequency but quite different wave numbers. So, in the general case, the electric field consists of potential and solenoidal parts

$$
\mathbf{E}=-\nabla \Phi-i \frac{\omega}{c} \mathbf{A}
$$

where $\mathbf{A}$ is the vector potential, $c$ is the speed of light. Hence, in our system of equations in general case appear three more unknown quantities, components of the vector potential $A_{x}, A_{y}$, and $A_{z}$. It means that we need to introduce three additional equations, which are obtained from the Maxwell equation

$$
\nabla \times \mathbf{B}=\frac{4 \pi}{c} e N_{0}\left(\mathbf{v}_{\mathbf{i}}-\mathbf{v}_{\mathbf{e}}\right)-i \frac{\omega}{c}\left(\nabla \Phi_{3}+i \frac{\omega}{c} \mathbf{A}\right) .
$$

Here $\mathbf{B}=\nabla \times \mathbf{A}$ is the magnetic field of perturbation, $\mathbf{v}_{\mathbf{i}}, \mathbf{v}_{\mathbf{e}}$ are the speeds of ions and electrons in the electric field given by Eq. (45).

As a result, we have to deal with the system of equations for the electric potential $\Phi_{3}$, components of the vector potential $A_{x}, A_{y}, A_{z}$, and perturbations of concentration $n_{e}, n_{i}$. It is convenient to use the following additional condition (Coulomb gauge):

$$
\nabla \cdot \mathbf{A}=0 .
$$

In the general case the system of equations is rather complicated. Due to this let us consider its solutions in the 2D case $\left(k_{y}=0\right)$ in the geometric optics approximation. Such systems of equations can be presented in the following form: 


$$
\begin{gathered}
{\left[\omega_{\mathrm{LHR}}^{2} k_{x}^{4} \rho_{e}^{2}-\left(\omega^{2}-\omega_{\mathrm{LHR}}^{2}\right) k_{x}^{2}\right]\left(\Phi_{3}-\frac{\omega}{k_{x} c} A_{x}\right)} \\
+\omega_{H e}^{2} k_{z}\left(k_{z} \Phi_{3}-\frac{\omega}{c} A_{z}\right)+i k_{x} \frac{\omega^{2} \omega_{H e}}{c} A_{y}=0, \\
{\left[k_{z}^{2}+\frac{\omega_{P i}^{2}}{c^{2}}-\frac{\omega^{2}}{c^{2}}\left(\frac{\omega_{P e}^{2}}{\omega_{H e}^{2}}+1\right)\right] A_{x}-i \frac{\omega_{P e}^{2} \omega}{c^{2} \omega_{H e}} A_{y}} \\
-\frac{k_{x}}{c}\left(\frac{\omega_{P i}^{2}}{\omega}-\frac{\omega_{P e}^{2} \omega}{\omega_{H e}^{2}}-\omega\right) \Phi_{3}=0, \\
{\left[k_{x}^{2}+k_{z}^{2}+\frac{\omega_{P i}^{2}}{c^{2}}-\frac{\omega^{2}}{c^{2}}\left(\frac{\omega_{P e}^{2}}{\omega_{H e}^{2}}+1\right)\right] A_{y}+i \frac{\omega_{P e}^{2} \omega}{c^{2} \omega_{H e}} A_{x}} \\
-i \frac{k_{x}}{c} \frac{\omega_{P e}^{2}}{\omega_{H e}} \Phi_{3}=0, \\
\left(k_{x}^{2}-\frac{\omega^{2}}{c^{2}}\right) A_{z}=\frac{\omega_{P e}^{2}}{c \omega}\left(k_{z} \Phi_{3}-\frac{\omega}{c} A_{z}\right)-\frac{\omega}{c} k_{z} \Phi_{3} .
\end{gathered}
$$

The first equation of this system describes the LH wave and the influence of the solenoidal electric fields on it. The remaining three equations correspond to electromagnetic (EM) waves interacting with the small-scale electrostatic wave. From the last Eq. (48) we find an approximate expression for the function $A_{z}$,

$$
A_{z}=\frac{\omega_{P e}^{2} c k_{z}}{\omega\left(\omega_{P e}^{2}+c^{2} k_{x}^{2}\right)} \Phi_{3}
$$

It follows from this equation that the longitudinal electric field

$$
k_{z} \Phi_{3}-\frac{\omega}{c} A_{z}=\frac{c^{2} k_{x}^{2}}{\omega_{P e}^{2}+c^{2} k_{x}^{2}} k_{z} \Phi_{3}
$$

for small scale perturbations $k_{x}^{2} \gg \omega_{P e}^{2} / c^{2}$ is determined by the electrostatic potential $\Phi_{3}$. For large-scale perturbations $k_{x}^{2} \ll \omega_{P e}^{2} / c^{2}$ the electromagnetic component $A_{z}$ causes significant decrease of the longitudinal electric field. It is convenient to find the components $A_{x}$ and $A_{y}$ from the second and the third Eq. (48) as the functions of the electrostatic potential $\Phi_{3}$. Assuming for simplicity that the wave frequency is close enough to the LHR frequency,

$$
\left|\omega^{2}-\omega_{\mathrm{LHR}}^{2}\right| \ll k_{z}^{2} c^{2} \frac{\omega_{P e}^{2}}{\omega_{H e}^{2}}
$$

we arrive at the following approximate system of equations instead of Eq. (48):

$$
\begin{gathered}
{\left[\rho_{e}^{2} k_{x}^{4}-k_{x}^{2}\left(\frac{\omega^{2}}{\omega_{\mathrm{LHR}}^{2}}-1\right)+\frac{M}{m} \frac{c^{2} k_{x}^{2} k_{z}^{2}}{c^{2} k_{x}^{2}+\omega_{P e}^{2}}\right] \Phi_{3}} \\
+\frac{\omega}{c}\left(\frac{\omega^{2}}{\omega_{\mathrm{LHR}}^{2}}-1\right) k_{x} A_{x}+i \frac{\omega_{H e}}{c} k_{x} A_{y}=0,
\end{gathered}
$$

$$
\begin{aligned}
& {\left[\left(k_{x}^{2}+k_{z}^{2}\right) k_{z}^{2}-\frac{\omega_{P e}^{4} \omega^{2}}{\omega_{H e}^{2} c^{4}}\right] A_{x}} \\
& =\frac{\omega_{P e}^{2}}{\omega_{H e} c}\left[\left(k_{x}^{2}+k_{z}^{2}\right) \frac{\omega^{2}-\omega_{\mathrm{LHR}}^{2}}{\omega \omega_{H e}}-\frac{\omega_{P e}^{2} \omega}{\omega_{H e} c^{2}}\right] k_{x} \Phi_{3}, \\
& {\left[\left(k_{x}^{2}+k_{z}^{2}\right) k_{z}^{2}-\frac{\omega_{P e}^{4} \omega^{2}}{\omega_{H e}^{2} c^{4}}\right] A_{y}} \\
& =i \frac{\omega_{P e}^{2}}{\omega_{H e} c}\left[k_{z}^{2}-\frac{\omega_{P e}^{2}}{\omega_{H e}^{2} c^{2}}\left(\omega^{2}-\omega_{\mathrm{LHR}}^{2}\right)\right] k_{x} \Phi_{3} .
\end{aligned}
$$

The left-hand sides of Eqs. (52) and (53) correspond to the whistler mode wave. Indeed, the terms in square brackets in the left-hand sides of Eqs. (52) and (53) coincide with the dispersion relation for the whistler mode wave. The link with the electrostatic LH wave is determined by the right-hand sides.

In the quasielectrostatic limit when the $k$-numbers of the excited waves are large enough,

$$
k_{x}^{2} \gg \frac{\omega_{P e}^{2}}{c^{2}}, \quad\left(k_{x}^{2}+k_{z}^{2}\right) k_{z}^{2} \gg \frac{m}{M} \frac{\omega_{P e}^{4}}{c^{4}},
$$

Eq. (51) reduces to

$$
\left[\rho_{e}^{2} k_{x}^{4}-k_{x}^{2}\left(\frac{\omega^{2}}{\omega_{\mathrm{LHR}}^{2}}-1\right)+\frac{M}{m} k_{z}^{2}-\frac{\omega_{P e}^{2}}{c^{2}}\right] \Phi_{3}=0 .
$$

The last term in square brackets in Eq. (54) describes the well-known EM correction and its influence on quasielectrostatic LH waves (see, e.g., Ref. 12).

The limiting case when $k_{z}=0$ should be considered separately starting from Eq. (48). It cannot be discussed correctly within the system of Eqs. (51)-(53) because some approximations were made while obtaining these equations. From the third equation in system (48) in the quasielectrostatic approximation we find the function $A_{y}$ in the following form:

$$
A_{y}=i \frac{\omega_{P e}^{2}}{\omega_{H e} c k_{x}^{2}}\left(k_{x} \Phi_{3}-\frac{\omega}{c} A_{x}\right) .
$$

After the substitution of Eq. (55) into the first Eq. (48) we arrive at the following equation for the combination $\Phi_{3}$ $-\omega / c k_{x} A_{x}$ :

$$
\left[\rho_{e}^{2} k_{x}^{4}-k_{x}^{2}\left(\frac{\omega^{2}}{\omega_{\text {LHR }}^{2}}-1\right)-\frac{\omega_{P e}^{2}}{c^{2}}\right]\left(\Phi_{3}-\frac{\omega}{c k_{x}} A_{x}\right)=0 .
$$

This equation describes small-scale LH perturbations along the $\mathrm{x}$-axis with the EM corrections.

The obtained above results allow us to investigate the mutual transformation of electrostatic and EM waves in the LH frequency range. First, let us find for which longitudinal wave numbers our previous results (the trapping of LH waves in $2 \mathrm{D}$ case) are valid in the presence of EM corrections. From Eq. (54) it follows that the electromagnetic corrections do not change significantly the results obtained in Sec. III, if 


$$
k_{z}^{2} \gg \frac{\omega_{P i}^{2}}{c^{2}} .
$$

In relation to the ionospheric modification experiments, our previous consideration is valid for $k_{z}$ numbers $\left|k_{z}\right| \gg 5$ $\times 10^{-6} \mathrm{~cm}^{-1}$. At the same time the characteristic $k_{z}$ numbers of the HF electromagnetic pump wave are of the order of $k_{z} \approx \omega_{P e} / c \sim\left(3 \times 10^{-4}-10^{-3}\right) \mathrm{cm}^{-1}$. It is clear that the excited UH and LH waves should have the longitudinal wave numbers of the same order of magnitude. In case of electrostatic bursts ("spikelets") in the upper auroral ionosphere we need to take into account that the plasma frequency and the averaged molecular weight of ions are significantly smaller than in the F-region. As a result for the heights $z$ $\sim 700-1000 \mathrm{~km}$ we obtain a similar condition $\left|k_{z}\right| \gg 5$ $\times 10^{-6} \mathrm{~cm}^{-1}$

Let us designate $k_{z}^{2}=Q \omega_{P i}^{2} / c^{2}$, where $Q$ is a coefficient which according to relation (57) should be large $Q>1$. It follows from Eqs. (52) and (53) that for the above mentioned $k_{z}$ numbers quasielectrostatic LH waves are able to excite propagating whistler mode waves with the frequencies of the order of the LHR frequency if $1<Q<\sqrt{M / m}$. Indeed, for such $k_{z}$ numbers the terms in square brackets in the left-hand sides of Eqs. (52) and (53) become equal to zero for the following $k_{x}$ numbers:

$$
k_{x}^{(0)}= \pm \sqrt{\frac{\omega_{P e}^{4} \omega^{2}}{\omega_{H e}^{2} c^{4} k_{z}^{2}}-k_{z}^{2}} .
$$

The relation (58) means that propagating whistler mode waves with the wave numbers $\left\{ \pm k_{x}^{(0)}, k_{z}\right\}$ can be excited by a localized source. To find the distribution of the whistler mode waves excited by the trapped LH oscillations in the Cartesian coordinates we need to proceed with Eqs. (52) and (53). Taking into account that they have similar solutions, let us discuss the solution of Eq. (53). In the 2D case such a solution takes the form

$$
\begin{aligned}
A_{y} \approx & i \frac{\omega_{P e}^{2}}{2 \omega_{H e} c k_{x}^{(0)}}\left[1-\frac{\omega_{P e}^{2}}{\omega_{H e}^{2}} \frac{\omega^{2}-\omega_{\mathrm{LHR}}^{2}}{c^{2} k_{z}^{2}}\right] \\
& \times \int \exp \left[-i k_{x}^{(0)}\left|x-x_{1}\right|\right] \frac{\partial \Phi_{3}}{\partial x_{1}} d x_{1} \\
& +i \frac{\omega_{P e}^{2}}{2 \omega_{H e} c k_{x}^{(0)}}\left[1-2 \frac{\omega_{P e}^{2}}{\omega_{H e}^{2}} \frac{\omega^{2}-\omega_{\mathrm{LHR}}^{2}}{c^{2} k_{z}^{2}}\right] \\
& \times \int \exp \left[-i k_{x}^{(0)}\left|x-x_{1}\right|\right] \frac{\delta n\left(x_{1}\right)}{N_{0}} \frac{\partial \Phi_{3}}{\partial x_{1}} d x_{1} .
\end{aligned}
$$

Here $k_{x}^{(0)}$ is the corresponding $k_{x}$ wave number for the propagating whistler mode wave, $k_{x}^{(0)} \approx \omega_{P e}^{2} \omega / \omega_{H e} c^{2} k_{z}$. Outside of a striation solution (59) describes two plane waves $\propto \exp \left[ \pm i k_{x}^{(0)} x\right]$ propagating in opposite directions along the $x$-axis from the trapped LH oscillations. According to Eq. (59) the amplitude of the EM field is a combination of two terms. The first integral describes freely propagating waves with the $\pm k_{x}^{(0)}$ wave numbers. The second integral appears if we take into account the interaction of the trapped LH waves with plasma irregularities $\delta n(x) / N_{0}$. Note that the trapped
LH oscillations are small-scale ones and hence the derivative $\partial \Phi_{3} / \partial x$ is large. Due to this the absolute value of such a term can exceed the absolute value of the first term.

Now let us briefly discuss the case when $k_{z}$ numbers are large enough $k_{z}^{2} \gg k_{c r}^{2}=(M / m)^{1 / 2} \omega_{P i}^{2} / c^{2}$. In this case the excited electromagnetic waves cannot propagate and they are localized near the LH wave packet. The corresponding distribution of these electromagnetic perturbations in the quasielectrostatic approximation takes the form

$$
\begin{aligned}
& A_{x}=-\frac{i \omega_{P e}^{4} \omega}{2 \omega_{H e}^{2} c^{3} k_{z}^{3}} \int_{-\infty}^{\infty} \exp \left[-\left|k_{z}\left(x-x_{1}\right)\right|\right] \frac{\partial \Phi_{3}}{\partial x_{1}} d x_{1}, \\
& A_{y}=\frac{\omega_{P e}^{2}}{2 \omega_{H e} c k_{z}} \int_{-\infty}^{\infty} \exp \left[-\left|k_{z}\left(x-x_{1}\right)\right|\right] \frac{\partial \Phi_{3}}{\partial x_{1}} d x_{1} .
\end{aligned}
$$

It is seen from the relations (60) and (61) that for the abovementioned $k_{z}$ numbers the EM component along the $x$-axis is significantly smaller than the component directed along the $y$-axis.

\section{DISCUSSION AND CONCLUSION}

In this paper we have discussed the possible trapping of LH waves in plasma depletions for two cases when thermal effects play an important role in trapping and when they are negligent. It should be mentioned that typical transverse sizes of perturbations in these two cases determined by Eqs. (10) and (19) are quite different. As the Larmor radius of the electron in the ionosphere is very small (of the order of $1-2 \mathrm{~cm}$ ) the corresponding $k_{x}$ numbers of the trapped $\mathrm{LH}$ waves are much larger when thermal effects are taken into account. In ionospheric modification experiments LH waves are excited due to decay of a strong UHR wave into another UHR wave with smaller frequency and LH wave. Both UHR waves are trapped in striations and have very small transverse sizes. This is confirmed by detection of UHR oscillations at oblique sounding with the help of VHF and UHF radars. ${ }^{20}$ Hence LH waves excited artificially in heating experiments also must have small transverse sizes. Because of this, thermal effects should be taken into account while discussing LH waves trapping in relation with the ionospheric modification experiments. In the upper auroral ionosphere the mechanism of excitation of LH waves in plasma depletions is quite different; linear transformation of large-scale whistler mode waves on plasma irregularities. Due to this and taking into account that natural plasma depletions have rather large transverse sizes $(20-50 \mathrm{~m})$, the trapping of $\mathrm{LH}$ waves can be explained based on the cold plasma approximation.

It should be mentioned that there is a slight similarity between the trapping of UHR and LH waves in striations. It was shown by Mjølhus that in reality UHR waves are quasitrapped. $^{22}$ There is a leakage from striations in the form of a large-scale electromagnetic slow extraordinary mode (the so-called Z-mode). As it was discussed in the present paper, the trapping of LH oscillations in striations can also be 
accompanied by the leakage of energy. But in the latter case the leakage exists in the form of a large-scale electromagnetic whistler mode wave. Due to high group velocity such artificially generated electromagnetic waves propagate for rather large distances and can be registered in the upper ionosphere.

In the ionospheric modification experiments UHR oscillations at the heater frequency appear due to conversion of the EM pump wave on small-scale irregularities (striations) via resonance (or thermal parametric) instability. These electrostatic UHR oscillations according to theoretical estimates have amplitudes substantially exceeding the amplitude of the pump wave. ${ }^{23,31}$ In the usual scheme with one wave pumping the excited LH waves should have a rather broad frequency spectrum and amplitudes significantly smaller than UHR oscillations at the heater frequency. Probably this is the reason why the LH waves were not detected before at oblique sounding in contrast with the UHR oscillations excited directly at the heater frequency. At the same time the results of the rocket experiment ${ }^{25-27}$ give a hint that $\mathrm{LH}$ waves are excited due to the heating. According to Kelley ${ }^{27}$ "The indicated wavelengths are so short that the lower hybrid drift mode may be the actual mode generated."

We have suggested a particular scheme of the ionospheric modification experiment in which the heating takes place simultaneously at two slightly different (of the order of the LHR frequency) frequencies $\omega_{1}$ and $\omega_{2}$. Our scheme provides the excitation of LH waves first of all at a given frequency $\omega_{3}=\omega_{1}-\omega_{2}$ with rather high intensity. The energy of such waves according to our analysis is not distributed in the whole heated volume but concentrated inside striations due to the trapping. Theoretical estimates in Sec. V show that relative plasma perturbations associated with trapped $\mathrm{LH}$ waves could achieve the values of the order of a few percent and have the transverse wave numbers $k_{x} \sim 0.2-0.02 \mathrm{~cm}^{-1}$. It means that such LH oscillations can probably be detected at oblique sounding by sensitive VHF or UHF radars. A special digital receiver similar to that one used recently in experiments utilizing oblique sounding in the HF range ${ }^{24,40}$ would be of prime importance. Time dependence of intensity and Doppler spectrum of the backscattered signal at the displaced frequencies $f_{r} \pm f_{\mathrm{LH}}$ are able to provide important information about physical processes in which LH waves take place. For example, the similarity of the Doppler spectrum at displaced frequencies $f_{r} \pm f_{\mathrm{LH}}$ and at the radar frequency $f_{r}$ would confirm that LH waves are trapped and move with striations. Also such measurements can demonstrate if the collapse of LH waves indeed exists at high pump powers in ionospheric modification experiments.

\section{ACKNOWLEDGMENTS}

This work is supported by the Leverhulme Trust Grant Reference No. F00185R.

${ }^{1}$ J. Labelle, P. M. Kintner, A. W. Yau, and B. A. Whalen, J. Geophys. Res. 91, 7113, DOI:10.1029/JA091iA06p07113 (1986)

${ }^{2}$ J. L. Vago, P. M. Kintner, S. W. Chesney, R. L. Arnoldy, K. A. Linch, T.
E. Moore, and C. J. Pollock, J. Geophys. Res. 97, 16935, DOI:10.1029/ 92JA01526 (1992).

${ }^{3}$ P. Kintner, J. Vago, S. Chesney, R. L. Arnoldy, K. A. Lynch, C. J. Pollock, and T. E. Moore, Phys. Rev. Lett. 68, 2448 (1992).

${ }^{4}$ A. I. Eriksson, B. Holback, P. O. Dovner, R. Boström, G. Holmgren, M. Andre, L. Eliasson, and P. M. Kintner, Geophys. Res. Lett. 21, 1843, DOI:10.1029/94GL00174 (1994)

${ }^{5}$ A. Vaivads, M. Andre, S. C. Buchert, J.-E. Walund, A. N. Fazakerley, and N. Cornilleau-Wehrlin, Geophys. Res. Lett. 31, L03804, DOI:10.1029/ 2003GL018142 (2004).

${ }^{6}$ J. Streed, C. Cattell, F. Mozer, F. Kokubun, and K. Tsuruda, J. Geophys. Res. 106, 6275, DOI:10.1029/2000JA900154 (2001).

${ }^{7}$ R. J. Strangeway, Adv. Space Res. 33, 1956 (2004).

${ }^{8}$ V. D. Shapiro, V. I. Shevchenko, A. S. Sharma, K. Papadopoulos, R. Z. Sagdeev, and B. B. Lebedev, J. Geophys. Res. 98, 1325, DOI:10.1029/ 92JA01729 (1993).

${ }^{9}$ D. Krauss-Varban, J. Geophys. Res. 94, 2537 (2004)

${ }^{10}$ K. McClements, J. J. Su, R. Bingham, J. M. Dawson, and D. Spicer, Sol. Phys. 130, 229 (1990).

${ }^{11}$ S. L. Musher and B. I. Sturman, JETP Lett. 22, 265 (1975).

${ }^{12}$ V. D. Shapiro, V. I. Shevchenko, G. I. Solov'ev, V. P. Kalinin, R. Bingham, R. Z. Sagdeev, M. Ashour-Abdalla, J. Dawson, and J. J. Su, Phys. Fluids B 53148 (1993).

${ }^{13}$ C. E. Seyler, J. Geophys. Res. 99, 19513, DOI:10.1029/94JA01572 (1994).

${ }^{14}$ A. V. Kostrov, A. V. Strikovskiy, and A. V. Shashurin, Plasma Phys. Rep. 27, 137 (2001).

${ }^{15} \mathrm{~S}$. Rosenberg and W. Gekelman, J. Geophys. Res. 106, 28867, DOI:10.1029/2000JA000061 (2001).

${ }^{16}$ A. Hedberg, B. Thidè, R. Boström, and R. Derblom, J. Geophys. Res. 89, 11038, DOI: $10.1029 / J A 089 i A 12 p 11038$ (1984)

${ }^{17}$ F. T. Djuth, M. P. Sulzer, and J. H. Elder, Geophys. Res. Lett. 21, 2725, DOI:10.1029/94GL01699 (1994)

${ }^{18}$ M. T. Rietveld, B. Isham, H. Kohl, C. La Hoz, and T. Hagfors, J. Geophys. Res. 105, 7429, DOI:10.1029/1999JA900476 (2000).

${ }^{19}$ P. F. Fialer, Radio Sci. 9, 923, DOI:10.1029/RS009i011p00923 (1974).

${ }^{20}$ J. Minkoff, P. Kugelman, and I. Weissman, Radio Sci. 9, 941, DOI:10.1029/RS009i011p00941 (1974).

${ }^{21}$ P. B. Rao, and G. D. Thome, Radio Sci. 9, 987, DOI:10.1029/ RS009i011p00987 (1974).

${ }^{22}$ E. Mjølhus, J. Plasma Phys. 29, 195 (1983).

${ }^{23}$ N. D. Borisov and T. R. Robinson, Phys. Lett. A 315, 126 (2003).

${ }^{24}$ J. M. Hughes, W. A. Bristow, R. T. Parris, and E. Lundell, Geophys. Res. Lett. 30, 2276, DOI:10.1029/2003GL018772 (2003).

${ }^{25}$ M. C. Kelley, T. L. Arce, J. Salowey, M. Sulzer, W. T. Armstrong, M. Carter, and L. Duncan, J. Geophys. Res. 100, 17367, DOI:10.1029/ 95JA00063 (1995).

${ }^{26}$ W. J. Peria, M. C. Kelley, and T. Franz, J. Geophys. Res. 104, 6797, DOI:10.1029/1999JA900027 (1999).

${ }^{27}$ M. C. Kelley, Geophys. Res. Lett. 31, L11806, DOI:10.1029/ 2004GL020105 (2004).

${ }^{28}$ H. L. Zhou, J. Huang, and S. P. Kuo, Phys. Plasmas 1, 3044 (1994).

${ }^{29}$ T. Leyser, Geophys. Res. Lett. 18, 408, DOI:10.1029/91GL00136 (1991).

${ }^{30}$ A. V. Gurevich, H. Carlson, A. V. Lukyanov, and K. P. Zybin, Phys. Lett. A 231, 97 (1997).

${ }^{31}$ A. V. Gurevich, A. K. Lykjanov, and K. P. Zubin, Phys. Lett. A 206, 247 (1995).

${ }^{32}$ N. Borisov and F. Honary, Phys. Lett. A 372, 2440 (2008).

${ }^{33}$ P. W. Schuck, C. E. Seyler, J. L. Pincon, J. W. Bonnell, and P. M. Kintner, J. Geophys. Res. 103, 6935, DOI:10.1029/97JA02922 (1998).

${ }^{34}$ P. W. Schuck, J. W. Bonell, and P. M. Kintner, IEEE Trans. Plasma Sci. 31, 1125 (2003).

${ }^{35}$ A. L. Akhiezer, I. A. Akhiezer, R. V. Polovin, A. G. Sitenko, and K. N. Stepanov, Plasma Electrodynamics (Pergamon, Oxford, 1975), Vol. 1, p. 414.

${ }^{36}$ Ya. N. Istomin and T. B. Leyser, Phys. Plasmas 5, 921 (1998).

${ }^{37}$ T. F. Bell and H. D. Ngo, J. Geophys. Res. 95, 141 (1990).

${ }^{38}$ D. R. Shklyar and I. Nagano, J. Geophys. Res. 103, 29515, DOI: 10.1029/ 98JA02311 (1998).

${ }^{39}$ N. Borisov, Phys. Lett. A 206, 240 (1995)

${ }^{40}$ J. M. Hughes, W. A. Bristow, and R. T. Parris, Geophys. Res. Lett. 31, L08808, DOI: 10.1029/2004GL019613 (2004). 
Physics of Plasmas is copyrighted by the American Institute of Physics (AIP). Redistribution of journal material is subject to the AIP online journal license and/or AIP copyright. For more information, see http://ojps.aip.org/pop/poper.jsp 